\title{
Prognostic Value of VEGF in Human Pancreatic Ductal Adenocarcinoma
}

\author{
Yun Jeong Lim, M.D.*, Jong Kyun Lee, M.D., Cheol Keun Park, M.D. ${ }^{\dagger}$, \\ Sang Yong Song, M.D. ${ }^{\dagger}$, Woo Young Jang, M.D. ${ }^{\ddagger}$, Hye Young Ha, \\ Dong II Park, M.D., Kyu Taek Lee, M.D., Seung Woon Paik, M.D., \\ Byung Chul Yoo, M.D. and Jong Chul Rhee, M.D. \\ Center for Health promotion*, Departments of Internal Medicine and Pathology ${ }^{\dagger}$, Samsung Medical Center, \\ Sungkyunkwan University School of Medicine, Seoul, Korea \\ Department of Pathology ${ }^{\ddagger}$, College of Medicine, Hallym University, Seoul, Korea
}

Background : Since pancreatic cancer metastasizes early regardless of the size of the primary tumor, it is suggested that angiogenic factor is upregulated in this disease. Among the angiogenic factors, vascular endothelial growth factor (VEGF) is the most potent and specific growth factor. The aim of this study is to elucidate the prognostic value of VEGF expression in pancreatic cancers.

Methods : We analyzed the VEGF expression using immunohistochemistry in 72 resected pancreatic ductal adenocarcinomas. We examined the prognostic value of the VEGF expression along with its relationship with the clinicopathological features.

Results : VEGF expression and mutant p53 expression were not associated with microvessel density. VEGF expression was positively associated with mutant p53 expression. There were no statistically significant relationships between the VEGF expression and other clinicopathological features, such as age, sex, CA19-9, tumor size, location, tumor differentiation, and stage. VEGF expression was not associated with patient survival.

Conclusion : VEGF expression was not associated with the microvessel density and patient survival in pancreatic ductal adenocarcinoma.

Key Words : VEGF, Pancreatic neoplasm, Prognosis

\section{INTRODUCTION}

Pancreatic cancer is one of the most common and lethal cancers in the world with a median survival time of approximately 3 months ${ }^{11}$. Although combined chemoradiation results in improvement of local control, it has only a modest impact on survival due to the development of distal metastases ${ }^{11}$. Pancreatic cancer can entail the substantial development of new blood vessels within the tumor tissue, and it is known that the growth and progression of solid tumors depend on such angiogenesis". Among the proangiogenic factors, the vascular endothelial growth factor (VEGF) is the most potent and specific growth factor ${ }^{3,4)}$. There are discrepancies about the prognostic value of the VEGF in pancreatic cancer. A previous report demonstrated that VEGF expression was an independent prognostic factor in pancreatic cancer ${ }^{5}$. There were somewhat contradictory results that the VEGF expression did not correlate with various clinicopathological parameters such as the vessel count, metastasis, recurrence and survival ${ }^{6)}$. To estimate the usefulness of the prognostic factor, many molecular and biological markers were investigated. This study was conducted to elucidate the prognostic value of VEGF expression as well as evaluate its relationship with clinicopathological variables in pancreatic ductal adenocarcinoma.

- Received : September 15, 2003

- Accepted : November 13, 2003

- Correspondence to : Jong Kyun Lee, M.D., Department of Internal Medicine, Samsung Medical Center, 50, Irwonbon-dong, Gangnam-gu, Seoul, 135-710, Korea Tel: 82-2-3410-3407, Fax:82-2-3410-3849, E-mail : jklee@smc.samsung.co.kr 
Table 1. The relationship between VEGF immunostaining and other clinicopathological features of pancreatic ductal adenocarcinomas (Spearman's rank correlation)

\begin{tabular}{|c|c|c|c|c|}
\hline & \multirow{2}{*}{ No. of cases } & \multicolumn{2}{|c|}{ VEGF staining } & \multirow{2}{*}{$p$ value } \\
\hline & & Negative & positive & \\
\hline Age (mean \pm S.D.) & 72 & $61.68 \pm 9.59$ & $56.95 \pm 13.81$ & \\
\hline \multicolumn{5}{|l|}{ Sex } \\
\hline Man & 43 & 28 & 15 & \multirow[b]{2}{*}{ NS } \\
\hline Woman & 29 & 21 & 8 & \\
\hline CA19-9 (mean \pm S.D.) & 72 & $54.16 \pm 12.5$ & $58.45 \pm 9.5$ & \\
\hline Tumor size (mean \pm S.D.) & 72 & $3.86 \pm 2.94$ & $4.28 \pm 5.14$ & NS \\
\hline \multicolumn{5}{|l|}{ Location } \\
\hline Head & 47 & 33 & 14 & \multirow{2}{*}{ NS } \\
\hline Body / tail & 25 & 16 & 9 & \\
\hline \multicolumn{5}{|l|}{ Tumor differentiation } \\
\hline Well & 14 & 6 & 8 & \multirow{3}{*}{ NS } \\
\hline Moderate & 44 & 34 & 10 & \\
\hline Poor & 14 & 9 & 5 & \\
\hline \multicolumn{5}{|l|}{ Stage } \\
\hline 1 & 13 & 6 & 7 & \multirow{4}{*}{ NS } \\
\hline 2 & 21 & 15 & 6 & \\
\hline 3 & 15 & 9 & 6 & \\
\hline 4 & 23 & 19 & 4 & \\
\hline
\end{tabular}

NS, not significant

\section{MATERIALS AND METHODS}

\section{Patients and tissue samples.}

Samples of 72 pancreatic ductal adenocarcinomas were obtained at the time of surgical resection from Samsung Medical Center (Seoul, Korea) between January 1995 and September 1999. The age of patients ranged from 16 to 79 years with a median age of 59.8 years. There were 43 male and 29 female patients. The median period of follow-up was 12.6 months (range, 1-66.2 months). The median survival time was 353 days. Follow-up data were gathered by reviewing of the patient's charts. The patients' outcomes were verified and updated through the medical record departments of Samsung Medical Center and the telephone. Fifty-six (77.8\%) patients died, and 16 patients were still alive during the follow-up periods. Twenty-six patients received adjuvant chemotherapy. Tissues were fixed in 10\% formalin for 12 hours and embedded in paraffin. Tissues were stained with hematoxylin and eosin. The histopathologic features of the pancreatic ductal adenocarcinoma were examined for the following: tumor size, location, tumor differentiation, and stage. The tumor stage was determined according to the staging manual of the American Joint Committee on Cancer ${ }^{7}$.

\section{Immunohistochemistry}

The representative tissue sections including both an adequate amount of tumor tissue and the adjacent non-tumor epithelial cells were selected and sectioned in $4-\mu \mathrm{m}$-thickness.
Immunohistochemical study was performed using the streptavin-biotin complex method. The primary antibodies used and working dilutions employed were as follows: VEGF (Santa Cruz Biotechnology, Inc, Santa Cruz, CA, USA, 1:2000), CD 34 (Immunotech, Marselle Cedex, France, 1:50), and p53 (Zymed, San Francisco, CA, USA, 1:80 dilution). For antigen retrieval, the sections were pretreated in an 800-W microwave oven in a $10 \mathrm{mM}$ citrate buffer of $\mathrm{pH} 6.0$ for 10 minutes. The sections were incubated with the primary antibody for 10 minutes, and then, the secondary antibody and the streptavin-peroxidase complex (LSAB kit, Dako, CA, USA) were applied, sequentially. DAB (3,3'-diaminobenzidine tetrahydrochloride) was used as a chromogen, and Mayer's hematoxylin counterstain was applied. Negative controls were run simultaneously with

Table 2. Cox proportional hazard model for Survival

\begin{tabular}{lc}
\hline \hline & $p$ value \\
\hline Age & NS \\
Sex & NS \\
CA 19-9 & NS \\
Size & NS \\
Location & NS \\
Tumor differentiation & NS \\
Stage & NS \\
CD34 & NS \\
Mutant p53 & NS \\
VEGF & NS \\
\hline
\end{tabular}

NS, not significant 
an omission of the primary antibody. The proportion of VEGF positive tumor cells was quantified and was assigned to one of two categories; $0,10 \%: 1,>10 \%{ }^{3}$. Microvessel density was recorded by counting the $\mathrm{CD} 34$ positive vessels in the highest vascularized area in X200 fields (a X10 ocular and a X20 objective (ens) $)^{8)}$. Vessels of a caliber larger than approximately eight RBCs, vessels with thick muscular walls, and vessels in sclerotic areas were excluded from the count. The counts were expressed as the total number of microvessels per $\mathrm{mm}^{2}$ in X200 fields using the image analysis system (CIRES program, IBAS model, Zeiss, Germany). Mutant p53 immunostaining was scored as follows. The proportion of positive tumor cells was quantified and was assigned to one of two categories; $0,10 \%: 1,>10 \%{ }^{\%}$. The stained slides were independently evaluated by two authors (W.O.J. and S.Y.S), and the difference in interpretation was resolved by a consensus.

\section{Statistical analysis}

The correlation between VEGF expression and clinicopathological features (age, sex, CA19-9, tumor size, location, tumor differentiation, stage, CD 34 and mutant p53) was determined by the Spearman's rank correlation. A Cox pro-
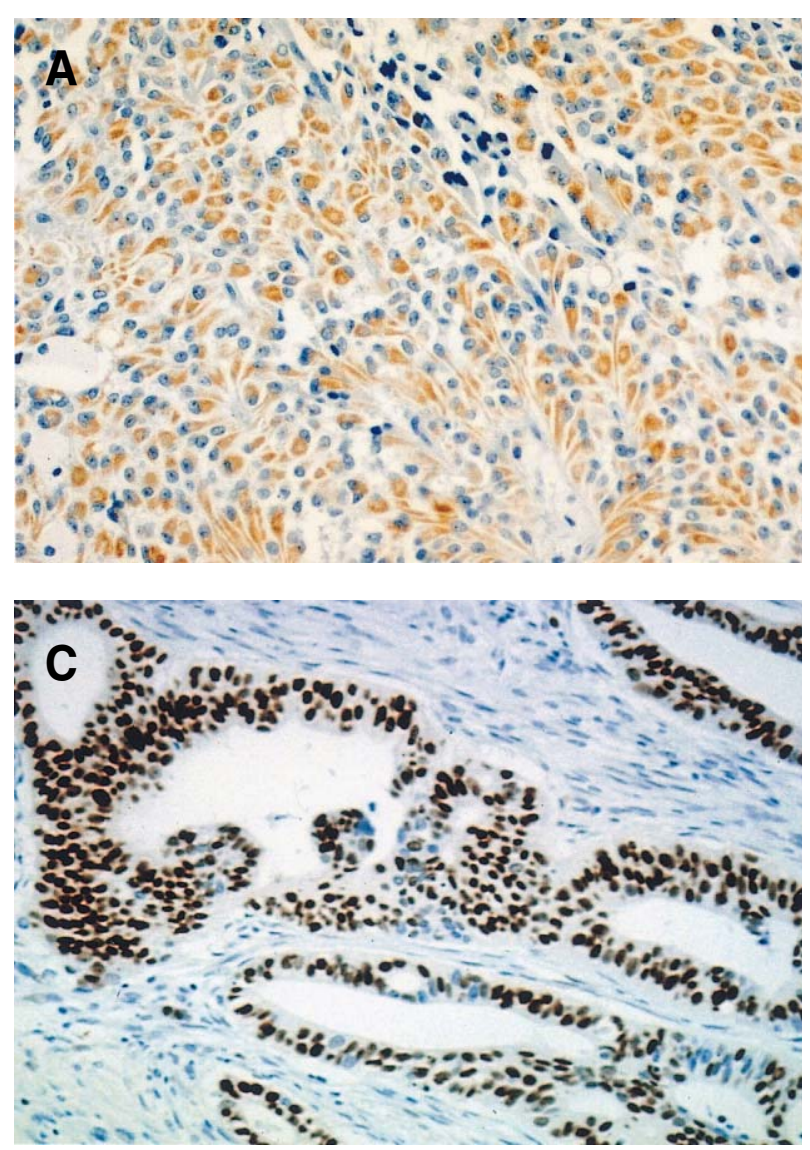

portional hazards model for the risk ratio was used to assess the simultaneous contribution of the following baseline covariates: age, sex, CA19-9, tumor size, location, tumor differentiation, stage, CD 34, mutant p53, and VEGF. Survival curves were constructed using the method of Kaplan-Meier. A probability of $p<0.05$ was considered statistically significant. RESULTS

VEGF immunoreactivity was present in $23 / 72$ tumors (32\%) (Figure 1A), and The mutant p53 expression was present in $44 / 72$ tumors (61\%) (Figure 1C). The VEGF expression was not associated with microvessel density $(p=0.059)$ (Figure 1B, 2), and microvessel density was not associated with the mutant p53 expression $(p=0.09)$. VEGF expression had a positive correlation with the $p 53$ mutation $(p=0.049)$. When the relationship between the VEGF expression and other clinicopathological parameters were evaluated, the statistical analysis revealed no significant correlation with other clinicopathological parameters (Table 1).

Multivariate analysis was performed according to the Cox proportional hazard model in order to evaluate the prognostic value of the VEGF expression, microvessel density, and mutant p53 expression. VEGF expression ( $p=0.239$ ), microvessel density $(p=0.398)$, and mutant $p 53$ expression $(p=0.772)$ did not predict

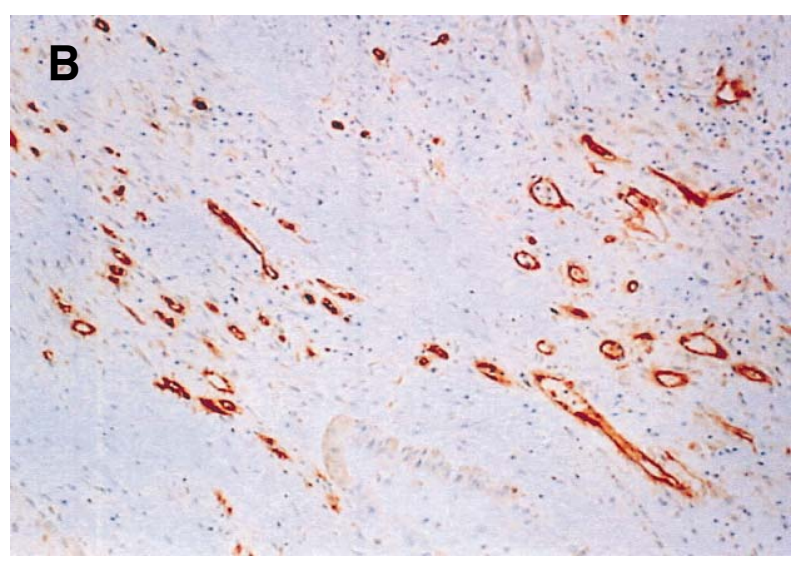

Figure 1. Immunohistochemical analysis (A) VEGF immunostaining of pancreatic ductal adenocarcinoma. Cytoplasms of the tumor cells are stained positive (H\&E, X200). (B) Immunostaining of $C D 34$ with high microvessel density (H\&E, X40). (C) Mutant p53 immunostaining of pancreatic ductal adenocarcinoma. The nucleuses of the tumor cells are stained positive (H\&E, X100). 


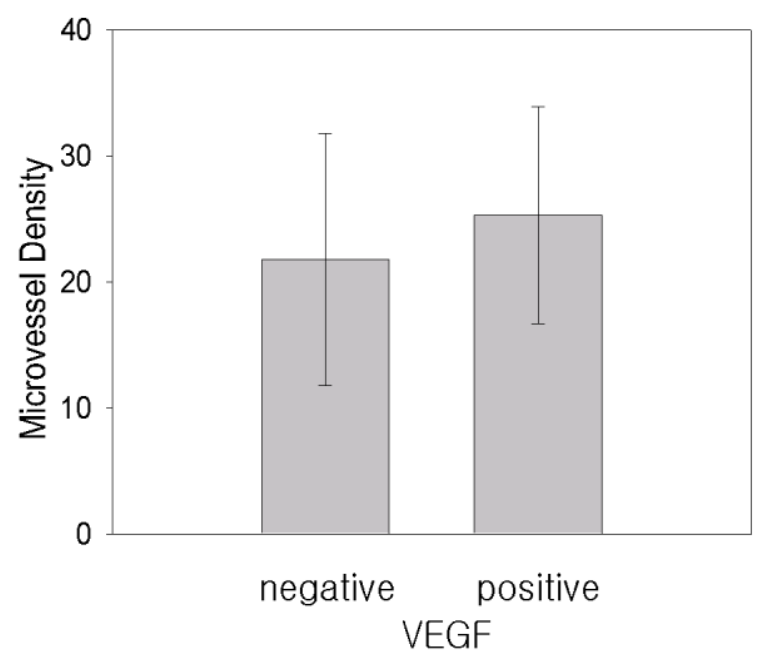

Figure 2. The correlation between VEGF expression and microvessel density. VEGF expression was not associated with microvessel density $(p=0.059)$.

unfavorable prognosis independently of other clinicopathological features (Table 2). The survival curves for the patients as a function of the VEGF expression are shown in Figure 3. It was illustrated using the Kaplan-Meier survival curve $(p=0.448)$ that VEGF expression was not associated with patient survival.

\section{DISCUSSION}

A reason for the poor prognosis in pancreatic cancer is the development of early metastasis regardless of the primary tumor growth". Angiogenesis is an integral part of the cascade of biologic events involved in tumor metastasis ${ }^{10)}$. If angiogenesis is essential for tumor growth and metastasis, there may be differences in the quantitative assessment of vascular proliferation between tumors with different prognostic features ${ }^{10,11)}$. Increased angiogenesis in pancreatic cancer is related to cancer aggressiveness ${ }^{2,5)}$. To date, many angiogenic factors for pancreatic cancers have been reported, such as the transforming growth factors (TGF)- $\alpha$, TGF- $\beta$, aFGF (acidic fibroblast growth factor), bFGF (basic fibroblast growth factor), angiogenin, VEGF, and PD-ECGF (platelet-derived endothelial cell growth factor) ${ }^{5,12,13)}$. Among the angiogenic factors, VEGF is the most potent and the most specific growth factor ${ }^{13)}$. Since pancreatic cancer metastasizes early regardless of the size of the primary tumor, it was expected that VEGF was upregulated in this disease ${ }^{3)}$. But, there are many controversies about the prognostic value of VEGF in pancreatic cancer. The previous report showed that strong VEGF immunoreactivity was present in the cancer ceu in $64 \%$ of the pancreatic cancer tissues. and the presence of VEGF was

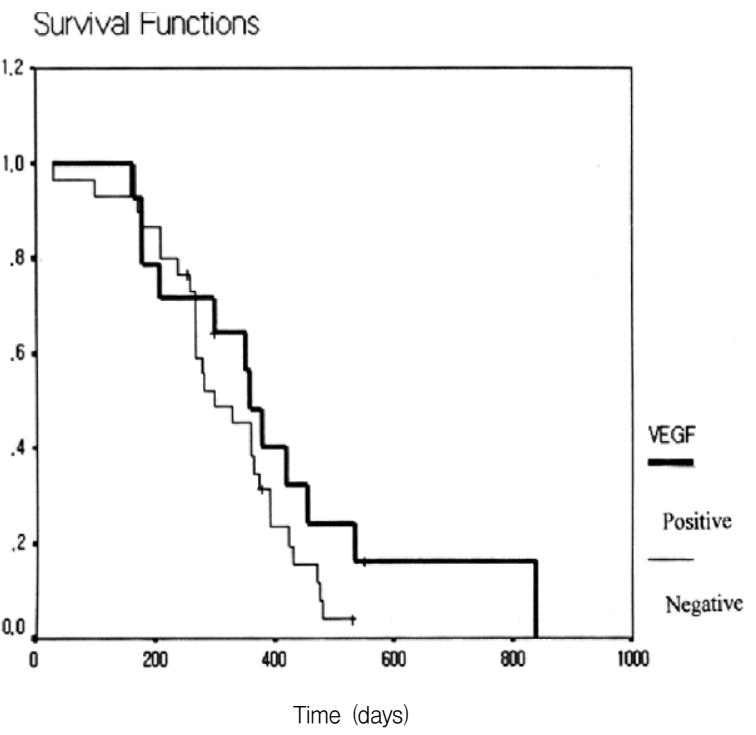

Figure 3. The effect of VEGF immunostaining on cumulative survival rate after the surgical resection of pancreatic ductal adenocarcinomas. Positive VEGF expression did not predict short survival $(p=0.448)$.

associated with an increased blood vessel number, large tumor size, and enhanced local spread but was not associated with a reduction in patient survival time ${ }^{3)}$. In our study, the VEGF expression was neither associated with an increased blood vessel number nor patient survival. Dilution, incubation time, and method of interpretation were somewhat different in the previous reports ${ }^{3-5,13)}$. Most of all, the analysis of the large sample size by immunohistochemitry combined with other objective experimental methods, such as Northern blot and real time PCR, was needed to elucidate the exact prognostic value of the VEGF in pancreatic ductal adenocarcinoma.

p53 mutations might be acquired in the later stages associated with metastatic progression and higher proliferation activity $^{14,15)}$. It was suggested that the mutant p53 gene was a potent stimulant of VEGF and was related to tumor angiogenesis ${ }^{14)}$. Mutant p53 expression may be a beneficial prognostic factor in pancreatic cancers ${ }^{9}$. . The previous report showed that mutations in the p53 gene occurred in approximately $60 \%$ of the tumors and appeared to be an independent prognostic factor for patient survival ${ }^{1,9)}$. The present study demonstrated that mutations in the p53 gene occurred in $61 \%$ of the tumors and was not a beneficial prognostic factor for patients with pancreatic cancer. The positive VEGF expression was associated with a poor prognostic factor such as mutant p53.

The only limitation of our study are the immunohistochemistric results. To know the exact prognostic value of VEGF, analysis of large sample size by immunohistochemistry, as 
well as the quantitative assay, such as the Northern blot, needs to be elucidated. The present study demonstrated that VEGF expression was neither associated with microvessel density nor patient survival in pancreatic ductal adenocarcinoma. This study suggests that it is difficult for the VEGF expression to be used as a prognostic marker in clinical practice.

\section{ACKNOWLEDGMENTS}

The authors thank Dr. Seonwoo Kim for the statistical analysis.

\section{REFERENCES}

1) van R i l JM , G accone G, P nedo HM . Pancreaticobilary cancer: the future aspects of medical oncobgy. Ann Oncol 10:296-299, 1999

2) Fo km an J.W hat is the evidence that tum ors are angingenesis dependent? J Natl Cancer hist 82:4-6, 1990

3) Itakura J, Ish iv ata T, Friess $\mathrm{H}, \mathrm{Fu}$ jii $\mathrm{H}, \mathrm{M}$ atsum oto $\mathrm{Y}$, Buch kr M W, Korc M . Enhanced expression of vascular endothelal grow th factor in hum an pancreatic cancer correhtes $w$ th bcal disease progression. C In Cancer Res 3:1309-1316, 1997

4) C bffey KP, Robinson GS. Regulation of VEGFNPF expression in tum or cells: consequences for tum or grow th and $m$ etastasis. Cancer Metastasis Rev 15:165-176, 1996

5) Ikeda N, Adachi M , Taki T, Huang C, H ashida H, Takabayashi A, Sho M, Nakajm a Y, Kanehiro H, H isanaga M, Nakano H, M yake M. Prognostic significance of angiogenesis in hum an pancreatic cancer. Br J Cancer 79:1553-1563, 1999

6) Fujm oto K, Hosotani R, W ada M, Lee JU, Koshba T, M yam oto Y, Tsuji S, Nakajm a S, Doi R, $\mathrm{m}$ am ura M. Expression of two angiggen $\dot{x}$ factors, vascular endothe lal grow th factor and phtekt- derived endothela 1 cell grow th factor in hum an pancreatic cancer, and its rehtionsh $\dot{p}$ to angiggenesis. Eur J Cancer 34:1439-1447, 1998

7) F em ing $\mathbb{D}$, Cooper JS, Henson DE, Hutter RVP, Kennedy BJ, M urphy GP, 0 Sullivan B, Sob in LH, Yabro JW . Am erican Joint Comm ittee On Cancer, Cancer Staging Manual. pp.121-126, Philadephí, Lippincott-Raven, 1997

8) W eidner N, Semple JP, W elch W R, Fo km an J. Tum or ang $\dot{D}^{-}$ genesis and $m$ etastasis: correhtion in invasive breast carcinom a. $N$ Engl J M ed 324:1-8, 1991

9) Barton CM, Staddon SL, Hughes CM, Hall PA, O Sullivan C, K ppel G, The is B, Russell RC, Neop to em os J, W illiam son RC. Abnom alites of the p53 tum our suppressor gene in hum an pancreatic cancer. Br J Cancer 64:1076-1082, 1991

10) 0 zer E, 0 zkal S, Karadem ir S, Sagol 0 , Sokm en S, Coker A, Kpeliogh A, Astarciblu I. Angiogenesis and p53 and H-ras mutations in pancreatic ductal adenocarciom a. Anal Q uant Cytol $H$ isto 121 :473-476, 1999

11) Shm oyam a $S$, Kam nishi M . Increased angiogen $\dot{n}$ express on $\dot{n}$ gastrix cancer correhted $w$ ith cancer progressin. $J$ Cancer Res C In Oncol 126468-474, 2000

12) Yam anaka Y, Friess H, Buch er M, Beger HG , U ch ida E, O nda M, Kobrn MS, Korc M. Overexpression of acid $\dot{x}$ and bas $\dot{x}$ fbroblast grow th factors $i$ hum an pancreatic cancer corre hates $w$ th advanced tum or stage. Cancer Res 53:5289-5296, 1993

13) $M$ neta $H, M$ ura K, $O$ g no T, Takebayashi $S, M$ isaw a $K$, Ueda $Y$, Suzuki I, D ictor M, Borg A, W ennerberg J. Prognostic value of vascular endothe lial grow th factor (VEGF) in head and neck squam ous cell carciom as. Br J Cancer 83.775-781, 2000

14) Bouvet M , E llis LM , N ishizaki M , Fu jw ara T, Li W , Bucana CD, Fang B, Lee JJ, Roth JA. Adenovius-m ediated will type p53 gene transfer downregulates vascular endo the lal grow th factor express in and inhbits angiggenesis in hum an cobn cancer. Cancer Res 58:2288-2292, 1998

15) Dam eron KM , Vo pert $0 \mathrm{~V}$, Tansky M A, Bouck N. The p53 tum or suppressor gene inhbits angiggenesis by stm $u$ ating the production of throm bospondin. Coll Spring Harb Sym p Q uant B D 159483-489, 1994 